\title{
DEVELOPMENT OF TEST BENCH FOR VARIABLE FREQUENCY DRIVE LOADING AND TESTING
}

\author{
Kirils Volosins, Kaspars Kroics \\ Riga Technical University, Latvia \\ kv.voloshin@gmail.com, kaspars.kroics@gmail.com
}

\begin{abstract}
The variable frequency drives today play an important role in industrial drives application. Set-up of frequency converter parameters, selecting the optimal working cycle and testing after maintenance or repair can be impossible or complicated in a field conditions. Therefore, a test bench that allows perform these operations in the laboratory could significantly reduce the testing costs and installation time. The paper describes design of the DC motor based test bench that emulates a real mechanical load for performing tests of variable frequency drive. After verification of system work in simulation software real hardware with the test bench control system was designed; the working principle of the bench and applied components is described in the paper. SCADA system was implemented to visualize the parameters of the test bench. The developed test bench could be used to test the variable frequency converter or test it at different load conditions. The experimental results are shown in the paper. The implemented measurement system allows to evaluate efficiency of different control algorithms. The example of industrial load emulation is shown in the paper.
\end{abstract}

Keywords: DC drive, frequency converter, electrical drive, test bench, SCADA.

\section{Introduction}

The variable frequency drives for induction and synchronous motors are used more and more often, as such approach allows efficiency improvement in many application fields. The motor itself usually can be operated for more than 20 years, but power electronics converter is not so reliable, therefore the variable frequency drive should be replaced or repaired if failure occurs. After repair work it is desired to test the variable frequency drive loading with a load similar as in the real conditions. As the test time usually is limited, some shorter tests are preferable to save the time. Some research papers, for example [1], offer load profiles for accelerated testing. One example of such load profile is shown in Fig. 1 [1]. By applying this proposed load profile, it is possible to test the electric drive faster. Even testing of the variable frequency drive with a nominal load for some minutes at the different speeds is enough to make a conclusion about possible failures. In both cases there is a need for a test bench that can provide more simple or advanced load profile to test the frequency converter after repair. The proposed test bench in this paper easily allows to implement such functionality.
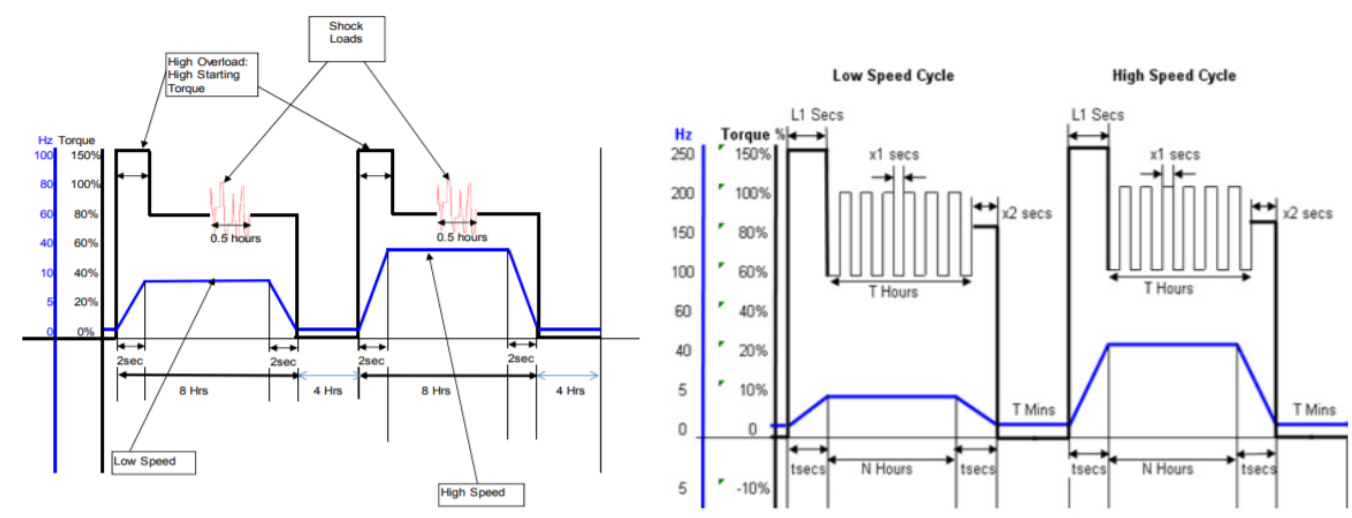

Fig. 1. Recommended torque and speed profile to test reliability of electrical drive

Also for testing of different control algorithms for later implementation it is needed to have a test bench that emulates load. Such test bench allows testing control algorithms that are implemented by using the programmable logic controller (PLC) or other digital or analogue method. By installing the energy meter, it is possible to measure the consumed electrical energy and find out the best control method. The paper describes the design of the DC motor based test bench that emulates a real mechanical load of industrial equipment, and therefore, is suitable very well for this application also.

In the research papers mainly test benches are developed in a way that the electrical machine under test and load emulating electrical machine are both controllers with the same control system, and therefore, it is much easier to implement more accurate and dynamic load testing. An electrical 
vehicle emulation test bench is described in [2; 3], a wave energy emulation system is shown in [4], a wind turbine - in [5], elevator load emulation is proposed in [6]. In the papers [7-9], control systems are shown that allow to implement accurate and dynamic load by controlling only the loading machine, but anyway an expensive torque sensor usually is used, or some complicated measurements from the motor under test are provided to implement control strategies. The goal of the test bench described in this paper is to provide a user friendly, easy for implementation loading system that could be used with variable frequency drives from different manufacturers and different power in some range, but do not concentrate on providing fully accurate and highly dynamic loading system.

Since for users of the variable frequency drive it is possible only to change the parameters of the outer speed control loop, which is slow, the proposed test bench also could be used for optimizing settings of this control loop. As the torque and speed control loop bandwidth of DC drive is limited, it is not possible to emulate full dynamics of real load behaviour, but for approximate tests the proposed test bench is valid.

\section{Operational principle of the test bench}

The electrical schematic of the test bench is shown in Fig. 2. Test bench is proposed mainly to test frequency converters and different control algorithms and control loop parameters of them, so it is desired that the induction motor is not replaced each time, although it is also possible with proper mechanical connection. The load emulator consists of a DC electrical machine and industrial 4quadrant DC drive that also regulates excitation current. The goal of the research is to develop a control system of the load emulator to imitate real industrial load.

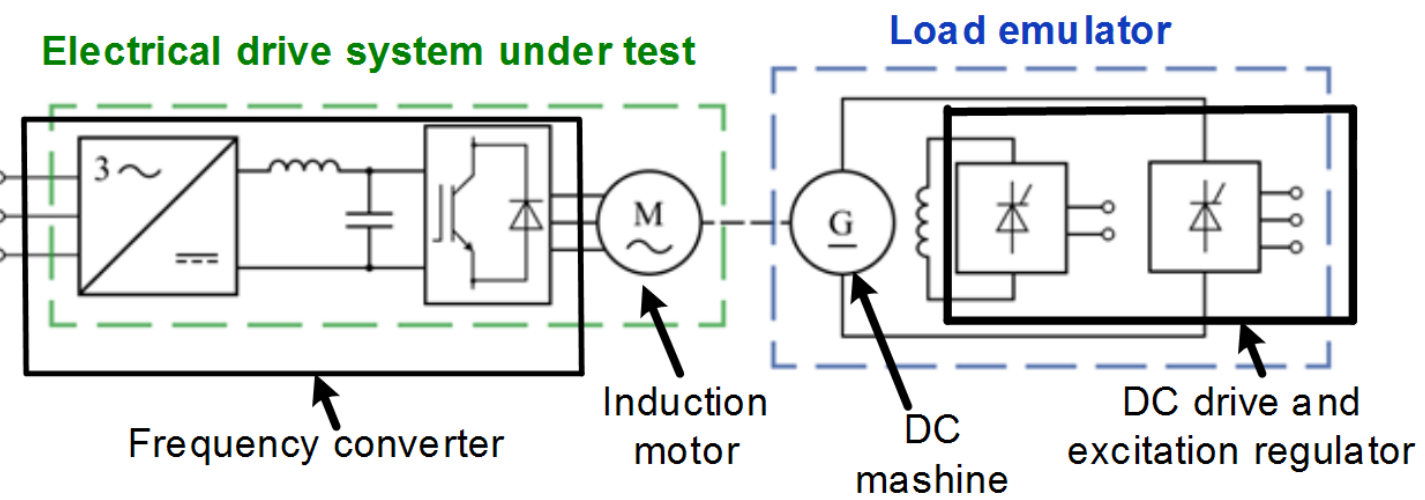

Fig. 2. Electrical schematic of the load emulation test bench

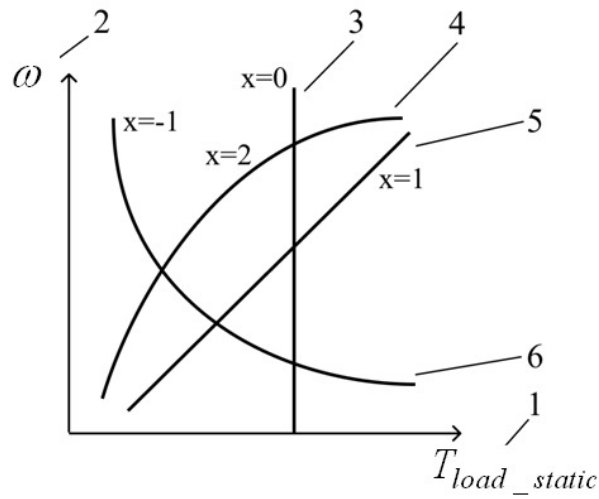

Fig. 3. Typical forms of torque/angular speed curve: 1 - static torque; 2 - angular speed; 3 - static torque is constant (not dependent on the change of frequency); 4 - static torque is a square function of

frequency; 5 - static torque is a linear function of frequency; 6 - constant static power

Loads of different driven machines can be represented with a torque/angular speed curves. They are used to determine a load torque, which a driven machine produces at supplied speed. Depending on the type of the load, there are various shapes of torque/angular speed curves. Typical forms of curves can be seen in Figure 3 [10]. All of these curves can be approximated with the help of 
mathematical equations; additionally for the dynamic process description inertia should be taken into account. So, Eq. 1 describes the load torque of industrial load:

$$
T_{\text {load }}=J \frac{d \omega}{d t}+T_{0}+a \cdot \omega+b \cdot \omega^{2}+c \cdot \omega^{3},
$$

where $T_{\text {load }}-$ load torque, $\mathrm{Nm}$;

$T_{0}$ - constant component of a static torque, $\mathrm{Nm}$;

$\omega$ - angular rotation speed of a motor, $\mathrm{min}^{-1}$;

$J$ - momentum of inertia;

$a, b, c$-coefficients, which describe the shape of the torque/angular speed curve.

If the speed change is slow, then derivative of the angular speed is very small and can be neglected, and the static load torque can be calculated as follows:

$$
T_{\text {load_static }_{\text {sti }}}=T_{0}+a \cdot \omega+b \cdot \omega^{2}+c \cdot \omega^{3} .
$$

The slow speed change can be done by implementing a slow speed regulator of the DC drive. In that case the static load, as shown in Figure 3, will be provided for the motor under the test. Such load is acceptable to test proper operation of the frequency converter after repair, or to measure the electrical energy consumption of different algorithms for industrial load control. The user can set the desired load by means of coefficients, or a user friendly interface can be developed to implement this setting in a visual manner. This simplification does not allow correctly emulate dynamics of the load, and theretofore for transient process modelling more complicated control of the load emulation system should be used.

The equation of movement of the test bench can be described by the following equation:

$$
T_{A C}-T_{D C}=\left(J_{D C}+J_{A C}\right) \frac{d \omega}{d t}+\left(D_{D C}+D_{A C}\right) \omega,
$$

where $T_{A C}$-torque of the induction motor,

$T_{D C}$ - torque of loading DC machine,

$D_{D C}, D_{A C}$ - friction coefficients of the DC machine and AC motor,

$J_{D C}, J_{A C}-$ momentums of inertia of the DC drive and AC motor.

Mechanical load on the shaft is provided and this torque can be calculated as follows:

$$
T_{\text {shaft }}=T_{D C}+J_{D C} \frac{d \omega}{d t}+D_{D C} \omega .
$$

The rotational speed of the mechanical shaft can be measured by using an encoder or tachometer, or determined with quite good accuracy from the mathematical model of the DC motor by measuring currents and voltages. The derivative of the rotational speed can be determined by storing some previous values of the speed and then calculating the speed change. The momentum of inertia $J_{D C}$ and the friction coefficient $D_{D C}$ of the DC machine can be determined by the method proposed in [11], or by other methods. In the same way the moment of inertia of the industrial load $J$ can be found in the datasheet of the mechanism or calculated, or determined by using experimental methods.

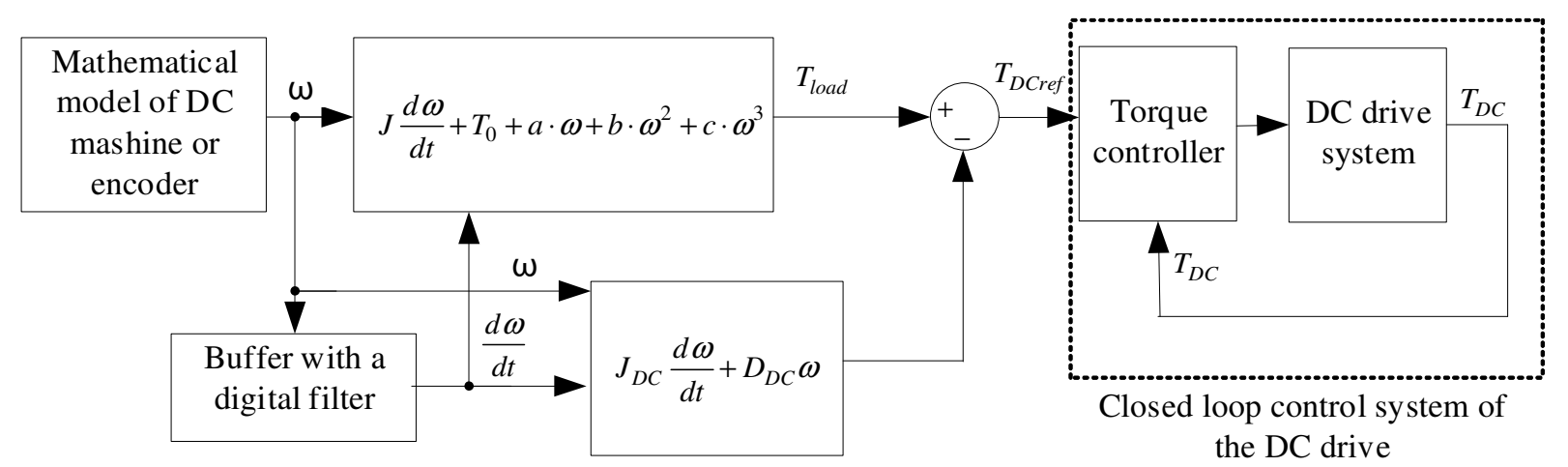

Fig. 4. Block diagram of control system to emulate dynamics of the load 
From Eq. 1, Eq. 3 and Eq. 4 it is possible to draw the control system block diagram to emulate dynamics of the load also. This block diagram is shown in Figure 4. As the input variable only the speed of the shaft is needed that in this case can be obtained from the industrial DC drive that calculates this value based on the motor mathematical model. This rotational speed is used to calculate the value of the emulating industrial load torque $T_{\text {load }}$, which can be described with Eq. 1 and the needed constants are the momentum of inertia $J$ and coefficients that describe the torque dependence on the angular rotational speed. From this torque $T_{\text {load }}$ should be subtracted the component that depends on the DC generator friction $D_{D C}$ and inertia of the DC machine $J_{D C}$, as the torque on the shaft cannot be controlled directly. The result is the reference torque to the DC drive $T_{D C \text { ref }}$. The control system of the DC drive via the closed loop system regulates the motor supplied to the DC machine in a way to keep the torque $T_{D C}$ equal to $T_{D C r e f}$. If the purpose of the test bench is to test dynamics of the frequency converter, then this control system should be with high dynamics to keep stability and emulate dynamics of the load accurately.

\section{Practical implementation of the test bench}

The previously described principle of operation was implemented into real hardware to create a system, which can be used to test operation of variable frequency drives. As far as the system will mainly be used in order to test repaired variable frequency drives, the main criterion of testing is whether the drive can run a motor under a certain load profile and dynamic load conditions. The idea is that a faulty variable frequency drive will not be able to provide certain torques (which fully functional drives are able to do), or provide proper speed or torque control dynamics, so the drive will be considered as faulty, if it cannot run a motor in a proper way.

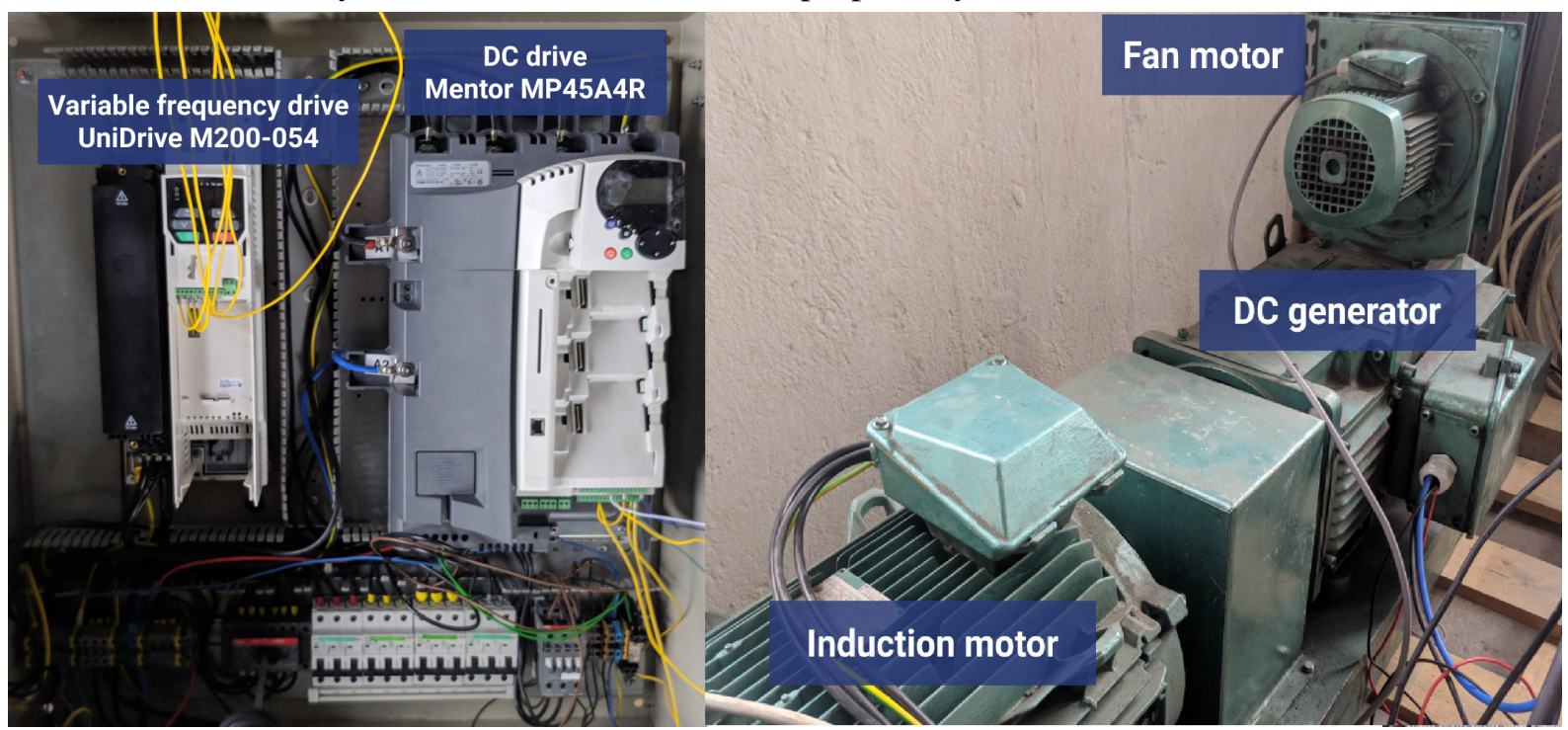

Fig. 5. Variable frequency drive test bench (communication cables are not connected)

A load torque will be provided by a DC generator/motor being run by the DC drive in torque control mode. That makes application of the test bench fast and universal, as finding and attaching real driven machines for testing purposes is not needed. The photo of the test bench is shown in Fig. 5. The $\mathrm{AC}$ induction motor and DC machine are mechanically coupled together and the DC motor/generator mainly acting in a generator mode provides the load torque to the $\mathrm{AC}$ motor. The $\mathrm{AC}$ induction motor is connected to the frequency converter and it is not difficult to replace the frequency converter by a new one to provide testing. The test bench consists of the following devices:

- variable frequency drive "Unidrive M200-054 00270A 11/15 kW";

- induction motor $(11 \mathrm{~kW})$;

- DC drive "Mentor MP45A4R $15 \mathrm{kW";}$

- DC generator $(17 \mathrm{~kW})$.

Both devices are configured for running corresponding motors. It can be done both with the help of built-in keyboards and special software ("Connect Drive Commissioning Software" for the variable 
frequency drive and "CTSoft" for the DC drive) [12;13]. If the configuration is done through the software, devices must be connected to the PC with the "Nidec Control Techniques USB to RS485 Converter".

For the DC generator to provide a certain load torque, the value of the DC drive parameter 4.11 (Torque mode selector) should be set to 2 (Torque control with speed override). Now, changing the parameter 4.08 (Torque reference \%) is going to change the corresponding load torque, if the rotation direction of the induction motor is reverse to the direction selected in the DC drive. To ensure that the rotation directions are set correctly, the induction motor and DC generator can be run separately. If the rotation directions are the same, there is a need of changing the rotation direction either in the settings of the variable frequency drive, or in the settings of the DC drive.

Load can be simulated by providing a load torque corresponding to the motor rotation speed. As far as the end version of the test bench does not require very high precision of the simulated load, the motor speed value can be taken from the estimated speed parameter of the drive. As it can be seen from Table 1, the variable frequency drive tends to output more precise speed values (based on comparison with the values shown by a digital tachometer).

Table 1

\section{Comparison of estimated speed parameters}

\begin{tabular}{|c|c|c|}
\hline $\begin{array}{c}\text { Speed shown by a digital } \\
\text { tachometer, } \text { min }^{-1}\end{array}$ & $\begin{array}{c}\text { Estimated speed of the } \\
\text { variable frequency drive } \\
\text { (parameter 5.004), } \text { min }^{-1}\end{array}$ & $\begin{array}{c}\text { Estimated speed of the DC } \\
\text { drive (parameter 5.04), } \text { min }^{-\mathbf{1}}\end{array}$ \\
\hline 603 & 607 & 660 \\
\hline 451 & 453 & 494 \\
\hline 300 & 301 & 332 \\
\hline
\end{tabular}

The simplest way of simulating the load is manually changing the "torque reference" parameter according to the current speed of the motor (from the torque/speed curve). Doing it manually might be reasonable, if the simulated load has a constant torque, but the more complicated the simulated loads are, the more time-consuming the manual torque reference change is. It is obvious that this task must be automated and the process itself can be improved.

Both "Unidrive M200" variable frequency drive and "Mentor MP" DC drive support Modbus RTU communication through RS-485 interface, so reading and writing their parameters can be done through serial communication. Torque reference can be changed either automatically (according to the current speed of the motor), or from a more user-friendly interface. Torque/speed curves of various loads can be entered by the user or calculated with the help of equation 1. Entered curves can be saved into the database to provide the ability of using the same load data in the following tests. Data from tests can also be saved into the database - user will have an option to analyse the data after the tests.

To build the system with required functionality, "Ecava Integraxor" software was used. It is used to configure and launch a SCADA system on a local server. It allows to configure communication with devices, create a connection to the database (in this project "PostgreSQL" database is being used) and custom data processing. The end-user interacts with the system through the web-interface. Pages shown to the user (user interface) are made manually meaning that the content could be customized according to the needs of the project.

\section{User interface description}

User can create a new load by either entering the points of a corresponding torque/speed curve or choosing the type of load and entering parameters of the load on the 'load configuration page' (webinterface).

To configure and start the test, "new measurement" page should be opened. It provides the following functionality:

- choosing the model of a variable frequency drive being tested;

- choosing the way in which the load torque will be set (it can be set either from a HTML slider or calculated automatically - from a previously entered torque/speed curve or from the equation); 
- choosing if data from the test will be stored in the database;

- starting and stopping the test;

- showing actual values of the measurement.

The content of the page is shown in Fig. 6 and will change, depending on the selected options, meaning that only required fields are shown during the process of measurement configuration. For example, if the user choses to store measurement data after the test, he will need to enter the unique name in the corresponding field. If the name has not been entered or it is not unique, the measurement will not be able to start and a corresponding information will be shown. If the measurement has been started, its values are shown in the table on the bottom of the page.

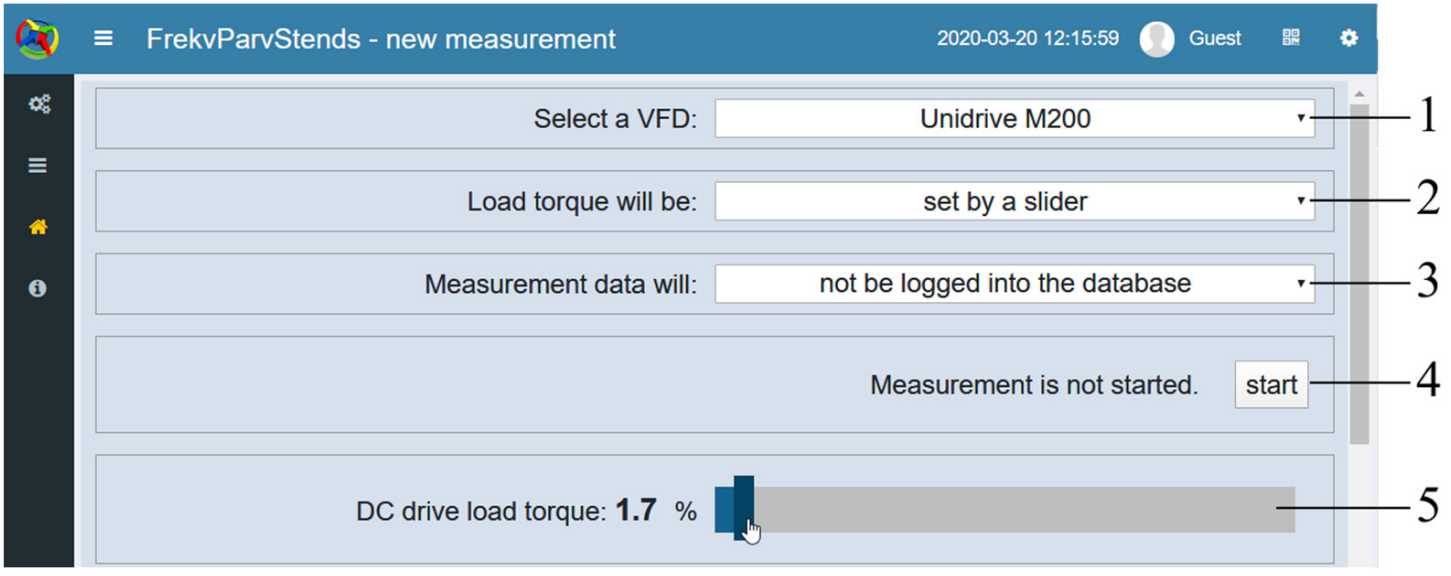

Fig. 6. New measurement page: 1 - selected variable frequency drive model; 2 - selected source of simulated load torque data; 3 - measurement data storage option selection; 4 - measurement state control button; 5 - slider for direct change of a DC drive torque reference parameter

In this version of the project measurement values are read from Modbus registers of the used drives. Depending on requirements for captured data and their precision, other measurement devices can be added to the system - rotational speed readings from the encoder, temperature of the motor or frequency converter. For the convenience of use, the measurement device needs to have a communication protocol supported by "Ecava Integraxor" software. If it does not have one, the interface must be changed - the input field must be added for the user to input the measured value.

\section{Load torque graph emulation example}

Figure 7 shows the user interface to enter the torque graph in an easy way. This graph was implemented to emulate industrial water pump load. The operation of the test bench was smooth and there were no large mechanical oscillations. Since the test bench does not have a torque sensor, it is not easy to make very precise conclusions about accuracy of the torque emulation and presence of small mechanical oscillations. But for testing of proper operation of the variable frequency drive test bench the torque was measured from the frequency converter screen. This torque is calculated from the induction machine mathematical model by measuring currents and voltages of the motor. Since the mathematical model of the induction machine is not fully precise, the displayed torque has some error, but nevertheless, these measurements allow conclude about suitable operation of the test bench.

Figure 8 shows the torque measurement results. At first the induction machine equipped with a frequency converter was operated without load and consumed power in a whole speed range fixed. This power represents lost power in bearings, brushes, etc. and should be subtracted from the emulated load curve. As it is shown in Fig. 8, this lost power should be compensated, as otherwise it creates a significant error. After compensation the measured torque from the frequency converter is almost equal to the emulated load graph allowing tests of the frequency converter with different profiles of industrial loads. 


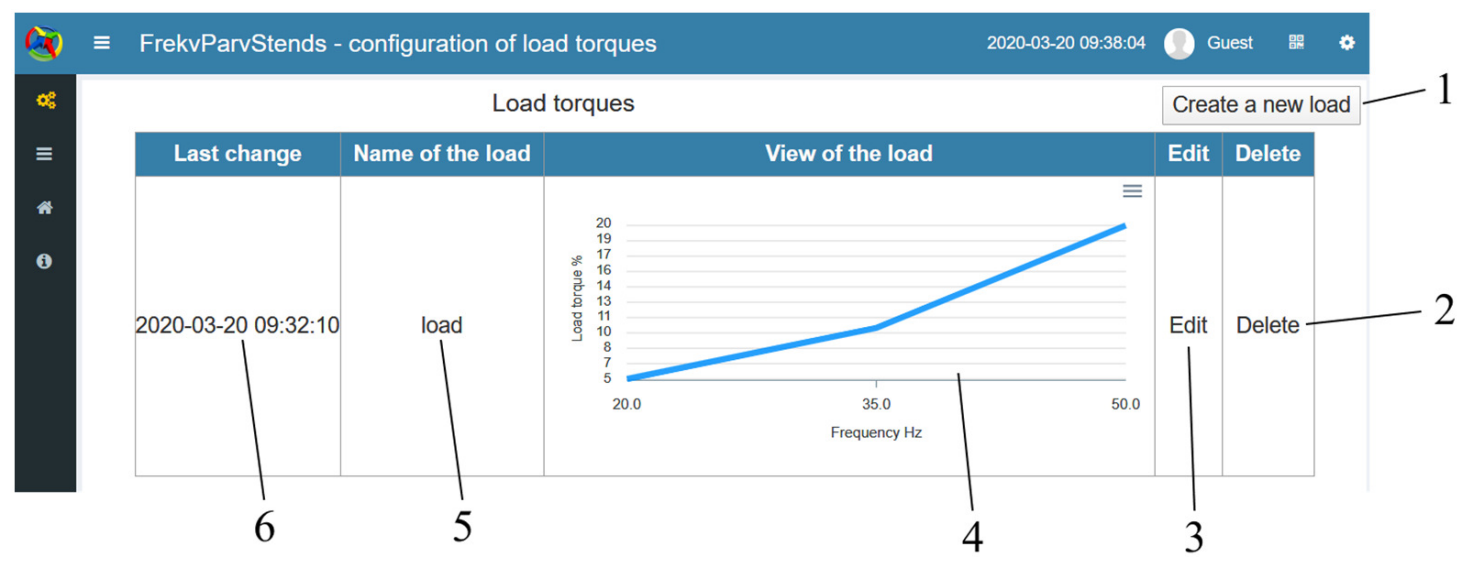

Fig. 7. Torque configuration page: 1 - button to create a new load; 2 - button to delete a load from the database; 3 - button to edit load values; 4 - characteristics based on the current values of the load; 5 - name by which the load is stored in the database; 6 - date of last changes of a load

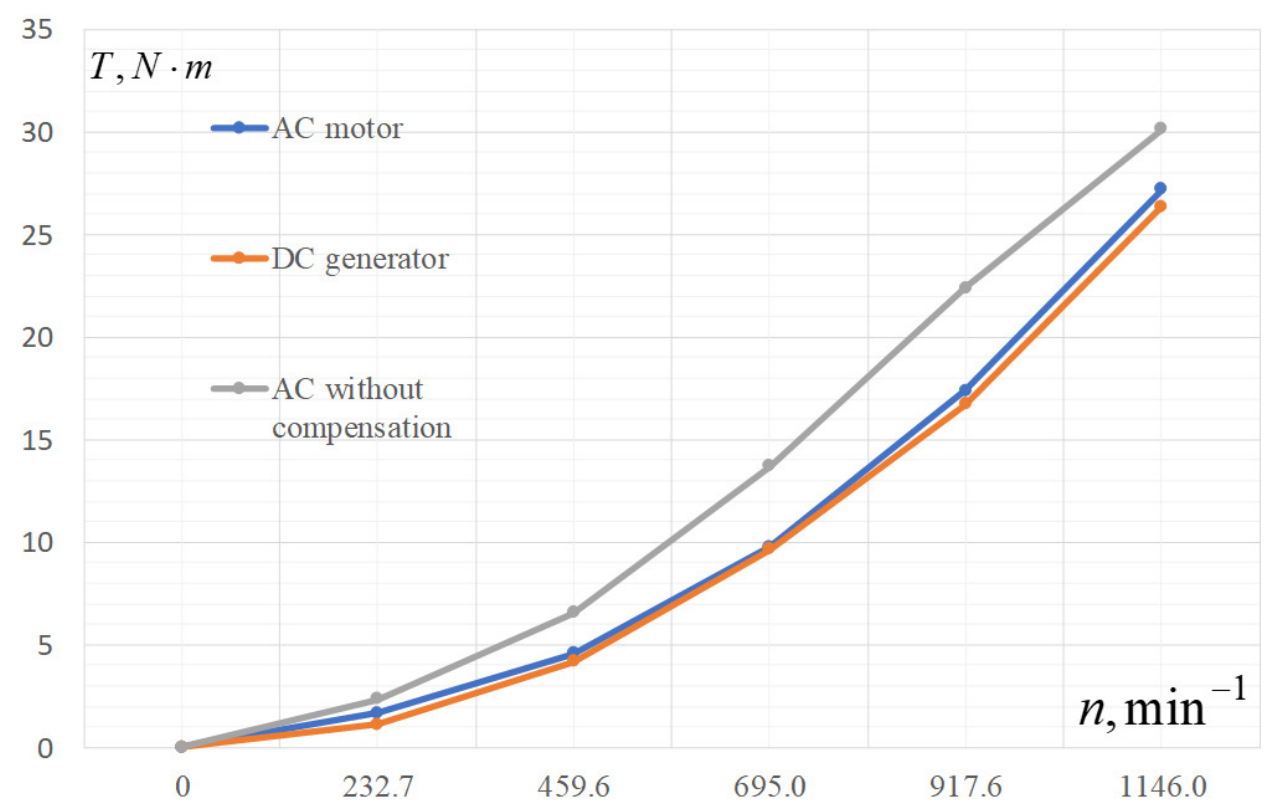

Fig. 8. Measured torque of both machines in case of exponential load emulation

\section{Conclusions}

The test of variable frequency drives and proper settings is needed quite often; the paper shows one possible development of the test bench for such application. In the paper shown mathematical equations are suitable to develop the control system for the industrial load emulating test bench. Loads of driven machines can be emulated using a DC drive that controls the DC electrical machine. "Ecava Integraxor" software allows build a user friendly interface to control such test bench. With the help of this software the data acquisition and processing functionality was built and tested. Experimental tests were provided and no oscillations were observed. Approximate torque measurement shows proper operation of the load emulation test bench. To evaluate the test bench accuracy and dynamic behavior a torque sensor should be installed and further tests provided. The test bench described in this paper is suitable for variable frequency drive testing purposes and can be used in applications, where there is a need to test behavior of variable frequency drives under mechanical load.

\section{Acknowledgements}

The authors would like to thank Dzintars Mednis for the provided ability to test the system on the hardware. 


\section{References}

[1] Yellamati D., Arthur E., James S., Morris G., Heydt T., Graf E. "Predictive Reliability Models for variable frequency drives based on application profiles," in 2013 Proceedings Annual Reliability and Maintainability Symposium (RAMS), Jan. 2013, pp. 1-6.

[2] Rodic M., Jezernik K., Trlep M. "Use of dynamic emulation of mechanical loads in the testing of electrical vehicle driveline control algorithms," in 2007 European Conference on Power Electronics and Applications, Sep. 2007, pp. 1-10.

[3] Brazis V., Kroics K., Grigans L. "Scientific laboratory platform for testing the electric vehicle equipped with DC drive," Latvian Journal of Physics and Technical Sciences, vol. 51, no. 5, 2014, pp. 56-64.

[4] Hazra S., Bhattacharya S. "Modeling and Emulation of a Rotating Paddle Type Wave Energy Converter," IEEE Transactions on Energy Conversion, vol. 33, no. 2, Jun. 2018, pp. 594-604.

[5] Yadav A. K., Singh M., Meena D. C. "Modelling and simulation of wind turbine emulator using DC motor," in 2016 IEEE 7th Power India International Conference (PIICON), Nov. 2016, pp. 15.

[6] Kroics K. "Development of Induction Motor Based Test Bench for Supercapacitor Braking Energy Recovery System Testing," in 2018 IEEE 59th International Scientific Conference on Power and Electrical Engineering of Riga Technical University (RTUCON), Nov. 2018, pp. 1-5.

[7] Rodic M., Jezernik K., Trlep M. "A feedforward approach to the dynamic emulation of mechanical loads," in 2004 IEEE 35th Annual Power Electronics Specialists Conference (IEEE Cat. No.04CH37551), Jun. 2004, vol. 6, pp. 4595-4601 Vol.6, doi: 10.1109/PESC.2004.1354812.

[8] Newton R. W., Betz R. E., Penfold H. B. "Emulating dynamic load characteristics using a dynamic dynamometer," in Proceedings of 1995 International Conference on Power Electronics and Drive Systems. PEDS 95, vol.1, Feb. 1995, pp. 465-470.

[9] Collins E. R., Huang Y. "A programmable dynamometer for testing rotating machinery using a three-phase induction machine," IEEE Transactions on Energy Conversion, vol. 9, no. 3, Sep. 1994, pp. 521-527.

[10]L. Ribickis and J. Valeinis, "Elektriskā piedziṇa mehatronikas sistēmās," Rīga RTU, vol. 286, 2008.

[11]Kroics K., Geidarovs R. "Practical Method for Experimental Detection of DC Motor Inertia to Design a Speed Regulator," in 2019 IEEE 7th IEEE Workshop on Advances in Information, Electronic and Electrical Engineering (AIEEE), Nov. 2019, pp. 1-6.

[12]"Mentor MP User Guide Issue 7." [online] [29.03.2020]. Available at: https://acim.nidec.com/drives/control-techniques/downloads/user-guides-and-software/mentormp.

[13]"Unidrive M200 Control User Guide Issue 3." [online] [29.03.2020]. Available at: https://acim.nidec.com/drives/control-techniques/downloads/user-guides-and-software/unidrive$\mathrm{m} 200$. 